\title{
Multi-Criteria Decision-Making Tool for the Safety of Bangladeshi Industries Using Fuzzy Logic
}

\author{
Muhammed Kamrul Islam ${ }^{1}$, Sajal Chandra Banik ${ }^{1}$, Nazmus Sakib ${ }^{1}$, Nurun Nahar Begum ${ }^{2}$ \\ ${ }^{1}$ Department of Mechanical Engineering, Chittagong University of Engineering and Technology, Chittagong, \\ Bangladesh \\ ${ }^{2}$ Department of Mechatronics and Industrial Engineering, Chittagong University of Engineering and \\ Technology, Chittagong, Bangladesh
}

\begin{abstract}
The paper focuses on the application of fuzzy logic as a Multi-Criteria Decision Making (MCDM) method to develop an idea of a managerial tool that can predict accidents in different industries. Accidents in industries are not directly dependent on the amount of expenses in different relevant sectors. Fuzzy logic enables us to quantify the opinion of experts with many years of experience and combine those quantified opinions with other parameter affecting the occurrence of accidents in industries which in this case are the amount of expenses in different relevant sectors. This combined assessment generates a relationship between the occurrence of accidents and the amount of expenses in different relevant sectors. The tool will help management to mitigate accidents which is more preferable than reacting to accidents.
\end{abstract}

Keywords: Fuzzy logic, HSE, Mamdani type, MCDC

*Corresponding Author

E-mail: kamrul.cuetme@gmail.com

\section{INTRODUCTION}

In developing countries like Bangladesh main focus is given on greater production. Owners take the regulatory compliances and associated expenses as constraining to productivity. The scenario is changing but not at a promising rate. The offshoring practices and outsourcing in workplaces of lower cost emerging countries have made a continuous and significant change (Chowdhury \& Tanim, 2016) [1]. Emphasis is being given now a day on occupational safety and environment to reduce accidents and casualties. To develop a reliable safety practice, attitude of the workers needs to be corrected by practicing good housekeeping and change in work culture in workplace. Accidents can be reduced by taking preventative actions through financing safety equipment, machinery, training the employees and process design (Beriha, Patnaik, Mahapatra, \& Padhee, 2012) [3]. There are many studies regarding workplace health and safety (WHS) but only a few used a dependable methodology to predict casualties. This paper emphasizes on the use of fuzzy logic as a Multi-Criteria Decision Making (MCDM) method to develop a tool for prediction of accidents in the Bangladeshi industries. With suitable risk identification model and risk control model accidents can be significantly mitigated. Though there exists lack of consensus regarding the selection of safety models to be used in risk identification it will be advantageous in building more applicable risk models using a different approach like fuzzy logic.

Safety performance measurement or risk management which is important for safety 
evaluation is one of the key tasks of Health and Safety Engineering (HSE) department. Organizations find it quite difficult because success results in the absence of an outcome (injuries or ill health) rather than a presence. This paper proposes a method to develop an effective tool that predicts the occurrence of injuries and accidents when there is insufficient data available. The significance of this study stems from the current lack of applicable models for analyzing data for HSE. Such model will help the management to understand the health and safety scenario of the industry. This will also enable them to make relevant decisions more precise and rapid too.

Not many researches have been done on safety performance using a fuzzy approach. Beriha et al. (Beriha et al., 2012) [3] introduced a model for safety performance analysis. This model predicts accidents in different sectors of industry accurately. Azadeha et al. (Azadeh, Fam, Khoshnoud, \& Nikafrouz, 2008) designed a fuzzy expert system for performance assessment of health, safety, environment (HSE) and ergonomics system factors in a gas refinery [2]. Joseph et al. (Tah \& Carr, 2000) proposed a methodology for assessing the risk exposure of a project. The parameters considered were quantity, cost, time and safety performance measures using fuzzy estimates of the risk components [8]. Zheng et al. (Zheng, Zhu, Tian, Chen, \& Sun, 2012) designed a fuzzy analytic hierarchy process (AHP) for safety evaluation using trapezoidal fuzzy numbers which gives early warning rating of the humid and hot environments [9-11]. To study work safety issue Dağdevirena and Yükselb (Dağdeviren \& Yüksel, 2008) applied the analytic hierarchy process (AHP) approach for simultaneous evaluation and multi-criteria as well [4]. This evaluation helps to define the faulty behavior risk (FBR) levels. Thus to prevent faulty behavior this approach would be helpful. Grassia et al. (Grassi, Gamberini, Mora, \& Rimini, 2009) introduced a model based on approximation to create a final rank of hazardous activities using fuzzy logic[5]. Tam et al. (Tam, Tong, Chiu, \& Fung, 2002) presented a modified Non-structural fuzzy decision support system (NSFDSS) which is appropriate for the evaluation of critical construction problems even if precise information is not available [9]. Mahdevaria et al. (Mahdevari, Shahriar, \& Esfahanipour, 2014) studied three underground mines in Kerman coal deposit and developed a dependable methodology using Fuzzy TOPSIS to identify the hazards and act accordingly in order to mitigate the risks before the accidents occur [7].

The objectives of the paper is to propose a method to develop a tool that predicts different types of accidents and injuries when sufficient relevant data are not available. It also includes categorizing accidents into diverse sets in order to have a better understanding of the underlying relations between injuries and expenses in the relevant sectors. The paper is quite unique in the sense that this is the only study conducted using fuzzy logic to analyze health and safety data of various industries in Bangladesh. The research of G.S. Beriha et al. (Beriha, et al., 2012) emphasized on different industries of India. The methodology they discussed did not use a varied set of data. Simultaneous use of data from different types of industries makes this paper more generic.

\section{METHODOLOGY}

Probability models used for quantification of risks and accidents using classic set theory cannot narrate risks in a significant manner. Shortage of relevant and precise data, uncertain association between cause and effect make it challenging to define a certain risk level using only traditional probability models. But experts with 
profound knowledge can provide a valuable judgment on risk assessment. Fuzzy logic uses these valuable judgments and explicitly considers the association between cause and effect.

\section{Fuzzy Inference System}

Fuzzy logic is an extension of multivalued logical system. In a broader sense it is basically synonymous with fuzzy sets, a theory that relates un-sharp boundaries to classes of objects. The membership in this theory is a matter of degree. Fuzzy logic is unique in the sense that, it can categorize an object in more than one exclusive set where levels of truth or confidence are different (Zadeh, 1965).

In a fuzzy set each point in the input space is mapped to membership value between zero and one. This is stated by the curve called Membership Function (MF). This function also defines the degree to which a given input belongs to a set (Zadeh, 1965) [10].

Two different types of data have been collected:

Type 1: The numbers of accidents and casualties of an industry. These data are further categorized into five categories:

(a) Accidents that do not cause any disability and do not involve any lost work days (ACC1). (b) Accidents that do not cause any disability but involve lost work days (ACC2).

(c) Accidents that cause temporary disability (ACC3).

(d) Accidents that cause permanent partial disability (ACC4).

(e) Accidents that cause permanent full disability or fatality (ACC5).

Type 2: Expenses in relevant sectors expressed as a percentage of the total revenue of annual budget. These data are further categorized into four different categories:

(a) Annual cost of health care.

(b) Annual cost of safety training.

(c) Annual cost of up-gradation of process related tools, instruments, machines, materials.

(d) Annual cost of safety equipment and tools.

Fuzzy logic uses expert opinion of different experienced persons. This mathematical tool can convert the linguistic knowledge into mathematical logics and combines it with the numerical data available. In this proposed method, a trapezoidal fuzzy number represents the fuzzy set " $A$ " which is defined by the quadruplet $(\mathrm{a}-\mathrm{d})$ shown in Figure 2 . The function becomes triangular membership function when $b=c$. Shown in Figure 3.

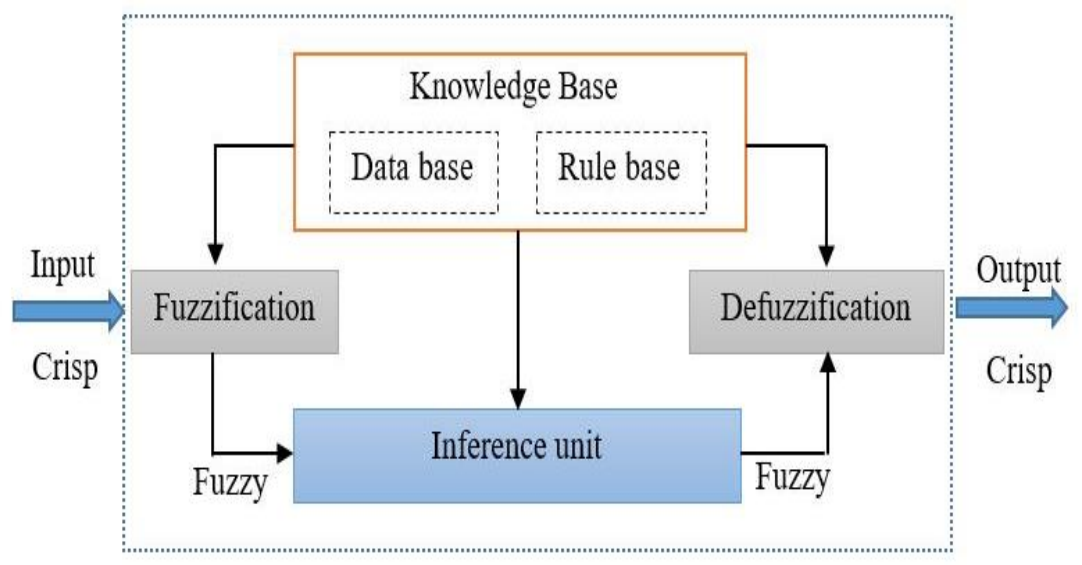

Fig. 1. Fuzzy inference system (Jamshidi, Yazdani-Chamzini, Yakhchali, \& Khaleghi, 2013) [6]. 


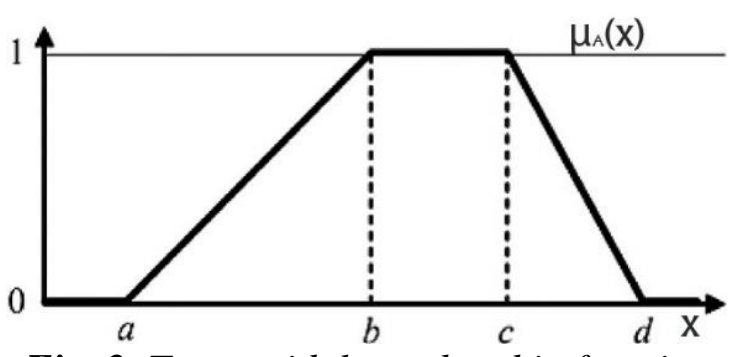

Fig. 2. Trapezoidal membership function (Beriha et al., 2012).

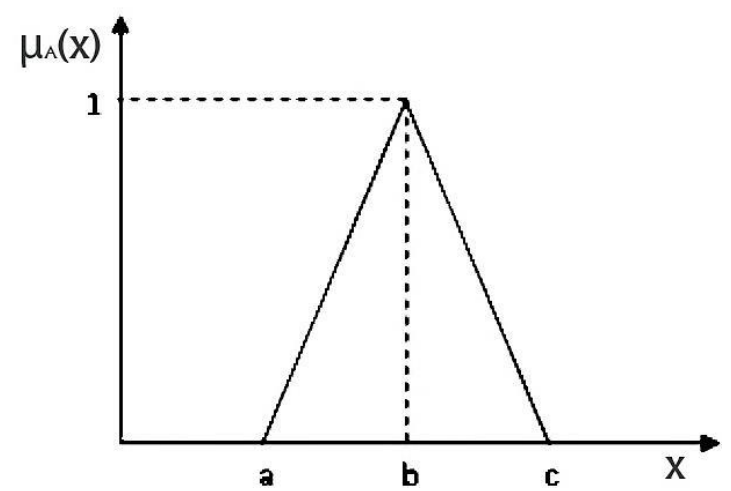

Fig. 3. Triangular membership function (Beriha et al., 2012).

Membership function $\mu_{A}(x)$ is defined as:

$$
\mu_{A}(x)=\left\{\begin{array}{cc}
0, & x<a \\
\frac{x-a}{b-a}, & a \leq x \leq b \\
1, & b \leq x \leq c \\
\frac{d-x}{d-e}, & c \leq x \leq d \\
0, & x>d
\end{array}\right.
$$

Fuzzy if-then rules are formed to get the output.

For a rule $R_{i}$ : If $x_{1}$ is $A_{1 i}$ and $x_{2}$ is $A_{2 i}, \ldots \ldots$, $x_{s}$ is $A_{s i}$ then $y_{i}$ is $C_{i} ; i=1,2, \ldots, M$

Where $M$ si total number of fuzzy rule, $x_{j}(j=1,2, \ldots . ., s)$ are input variables, $y_{i}$ are the output variables, and $A_{j i}$ and $C_{i}$ are fuzzy sets modeled by membership functions $\mu_{A j i}\left(x_{j}\right)$ and $\mu_{c i}\left(y_{i}\right)$, respectively.

The aggregated output for the $M$ rules is: $\mu_{c i}\left(y_{i}\right)=\max \left\{\min \left[\mu_{A 1 i}\left(x_{1}\right), \mu_{A 2 i}\right.\right.$ $\left.\left.\left(x_{2}\right), \ldots \ldots \ldots, \mu_{A s i}\left(x_{s}\right)\right]\right\} ; I=1,2,3 \ldots ., M$

To have a clear understanding of industrial health and safety scenario, different industries of EPZs were visited. As expert opinion is one of the most important parameters in Mamdani interface system the model has been reviewed by different experienced and expert personnel of HSE sectors to ensure the identification of most accurate input and output parameters. Type 1 data are used as the input parameters. Type 2 data are used as output parameters.

\section{RESULT AND DISCUSSION}

The paper focuses on predicting numerical value of accidents occurring in different industries of Bangladesh as a means of measuring the occupational health and safety of the respected industries. The range of membership functions in input and output parameters with their definition are shown in Tables 1 and 2, respectively. Figure 4 represents the fuzzy model of Mamdani type used for the analysis. A sample input membership function is shown in the Figure 5. Figure 6 represents a sample output membership function.

A holistic view of the company's health and safety condition can be achieved by aggregating risks models based on fuzzy set theory. The rule-based system is created by aggregating and analyzing the opinions of experts and combining them with the information which explain the dependence, linkage, and relationships among modeled factors. The factors in this case are the input and output data used for the modeling. The rules are generally a set of if/then control rules. 17 rules are proposed in this paper to acquire desired output of statistical data of accidents in different industries in Bangladesh. One of the rules is given below:

If (health care is high) and (safety training is high) and (safety tools is low) and (safety equipment is high) then (ACC1 is safe) (ACC2 is) (ACC3 is very safe) (ACC4 is safe) (ACC5 is safe). 
Figure 7 shows all the rules and Figure 8 is the rule base obtained after cognitive intervention. Figure 9 represents a sample surface view. After defuzzification process the desired data are attained which exhibit the number of accidents in the industries. Tables 3-7 show the numerical values (predicted and actual) of accidents acquired from the model. The actual numbers of accidents are listed in column 2 while column 3 shows the predicted values. The percentage errors are listed in column 4 which indicates whether the predicted value is more or less than actual numeric value of the accidents. The fifth column specifies the residual.

From Tables $3-7$ it is seen that the model predicted more number of accidents than actual in the industries 4, 7, 10 and 9 for the accident types ACC1, ACC2 and ACC4 while it predicted less than the actual number in the industries 1, 6 and 9 for the accident types ACC1, ACC3, ACC4 and ACC5. Four out of ten cases in ACC5 type accident which indicates the fatality shows no occurrence as it is a rare phenomenon.

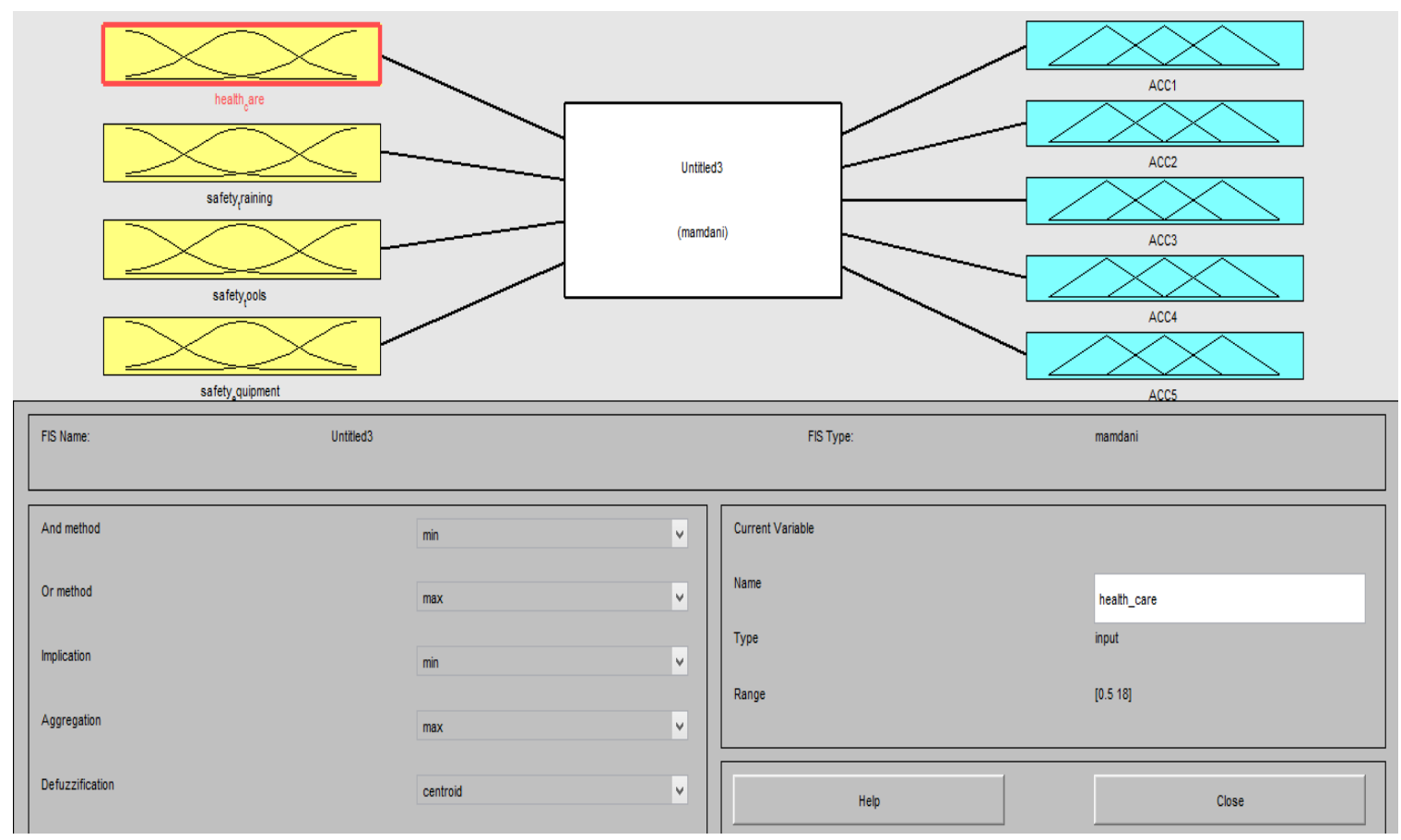

Fig. 4. Mamdani type fuzzy model.

Table 1. Range of membership functions in output parameters.

\begin{tabular}{|l|l|c|c|c|c|c|}
\hline Linguistic value & Definition & ACC1 & ACC2 & ACC3 & ACC4 & ACC5 \\
\hline Extremely unsafe & $\begin{array}{l}\text { Very high } \\
\text { rate of } \\
\text { accident }\end{array}$ & $1,1,10.8$ & $3,3,11.4$ & $1,1,4.8$ & $0.2,0.2,0.56$ & $0,0,0.04$ \\
\hline Very unsafe & $\begin{array}{l}\text { High rate } \\
\text { accident }\end{array}$ & $7.533,10.8,17.33$ & $8.56,11.4,17$ & $3.53,4.8,7.33$ & $0.44,0.56,0.81$ & $0.03,0.04,0.067$ \\
\hline Unsafe & $\begin{array}{l}\text { Lower } \\
\text { than high } \\
\text { rate of } \\
\text { accident }\end{array}$ & $14.2,20.6,30.4$ & $14.21,19.8,28.2$ & $6.07,8.6,12.4$ & $0.68,0.92,1.28$ & $0.05,0.08,0.12$ \\
\hline Safe & $\begin{array}{l}\text { Low rate } \\
\text { of } \\
\text { accident }\end{array}$ & $21.51,31.57,43.03$ & $21.19,31,39.41$ & $9.23,13.67,17.47$ & $0.98,1.4,1.76$ & $0.08,0.13,0.17$ \\
\hline Highly safe & $\begin{array}{l}\text { Negligible } \\
\text { rate of } \\
\text { accident }\end{array}$ & $35.61,50,50$ & $31,45,45$ & $13.7,20,20$ & $1.4,2,2$ & $0.13,0.2,0.2$ \\
\hline
\end{tabular}


Table 2. Range of membership functions in input parameters.

\begin{tabular}{|l|l|c|c|c|c|}
\hline $\begin{array}{c}\text { Linguistic } \\
\text { value }\end{array}$ & \multicolumn{1}{|c|}{ Definition } & Cost of health care & $\begin{array}{c}\text { Cost of safety } \\
\text { training }\end{array}$ & $\begin{array}{c}\text { Cost of upgradation } \\
\text { of tool }\end{array}$ & $\begin{array}{c}\text { Cost of safety } \\
\text { equipment }\end{array}$ \\
\hline Low & $\begin{array}{l}\text { Least expense per } \\
\text { year }\end{array}$ & $0.5,0.5,5.856$ & $0.5,0.5,3.21$ & $0.8,0.8,12.48$ & $0.8,0.8,5.34$ \\
\hline Medim & $\begin{array}{l}\text { Average expense per } \\
\text { year }\end{array}$ & $2.28,7.64,10.86,16.21$ & $1.18,2.76,6.61$ & $3.58,16.61,27.85$ & $1.94,6.48,11.02$ \\
\hline High & $\begin{array}{l}\text { High level of } \\
\text { expenses per annum }\end{array}$ & $12.64,18,18$ & $4.572,7.286,10$ & $18.77,30,30$ & $7.62,15,15$ \\
\hline
\end{tabular}

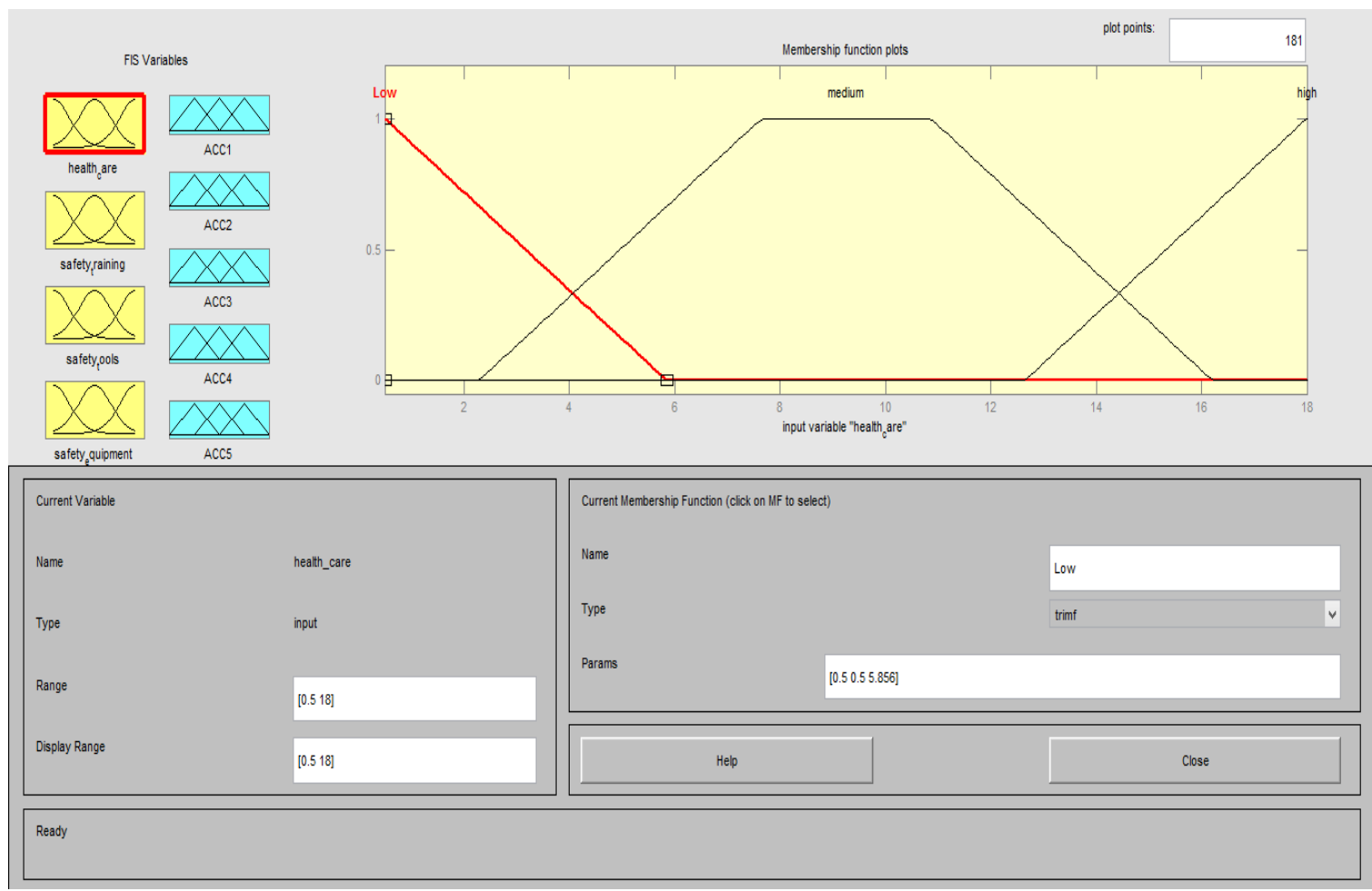

Fig. 5. Input membership function for annual cost of health care.

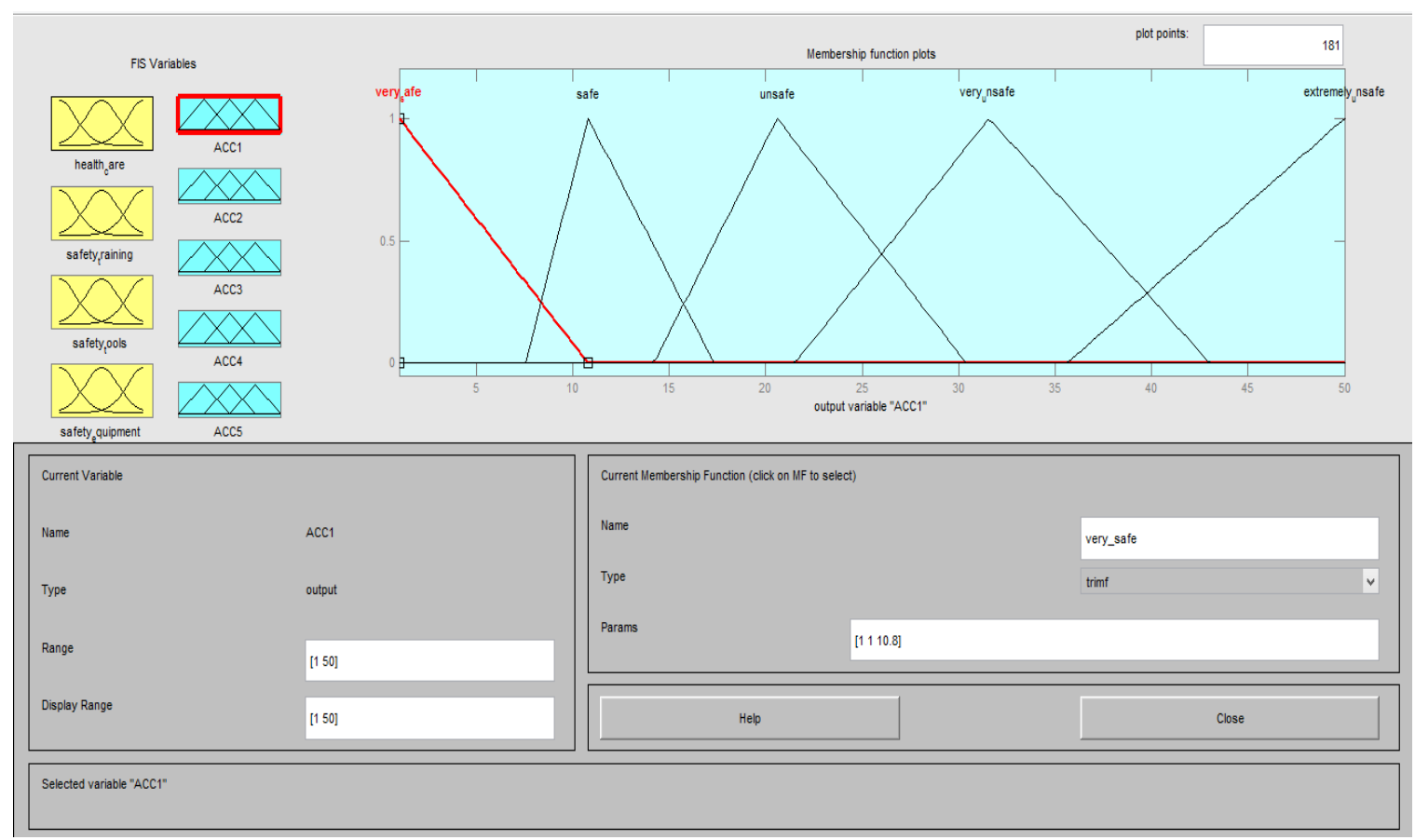

Fig. 6. Output membership function (ACC1). 


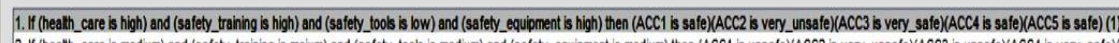

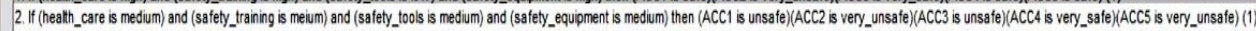

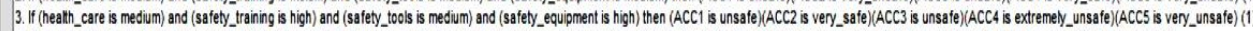

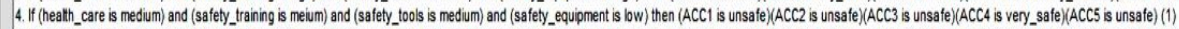

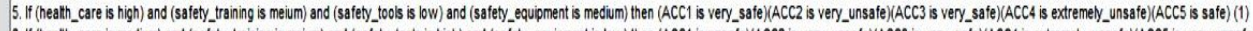

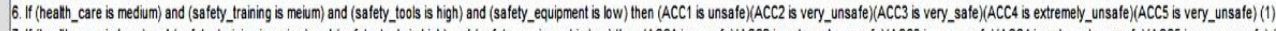

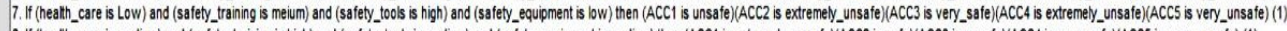

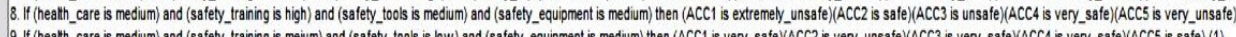

10.If (heath, care is medium) and (safety_traning is low) and (safety_tools is medium) and (safery_equipment is low) then (ACC1 is unsate)(ACC2 is very_safe) (ACC3 is extremer__unsafe)(ACC4 is extremery__unsafe)(ACC5 is very_unsafe) (1)

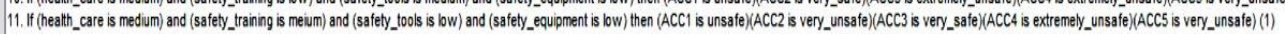

121 (heation

13.1 (hestht

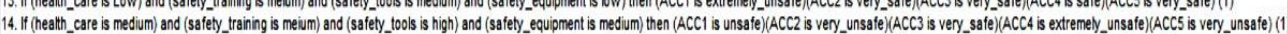

15. (5eä__

(1)

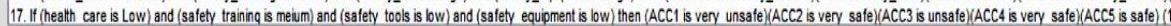

hesth_care is

satery_traning is

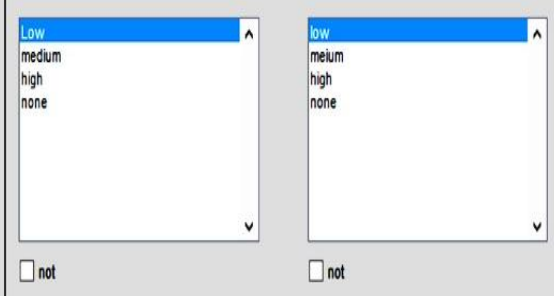

satety_tools is

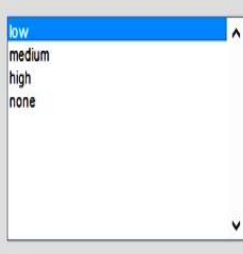

$\square$ not and

safery_equipmentis

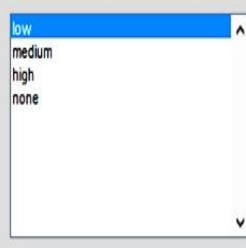

○not
Then

\section{ACC1 is}

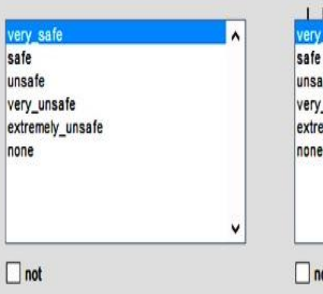

Fig. 7. Fuzzy if then rules.

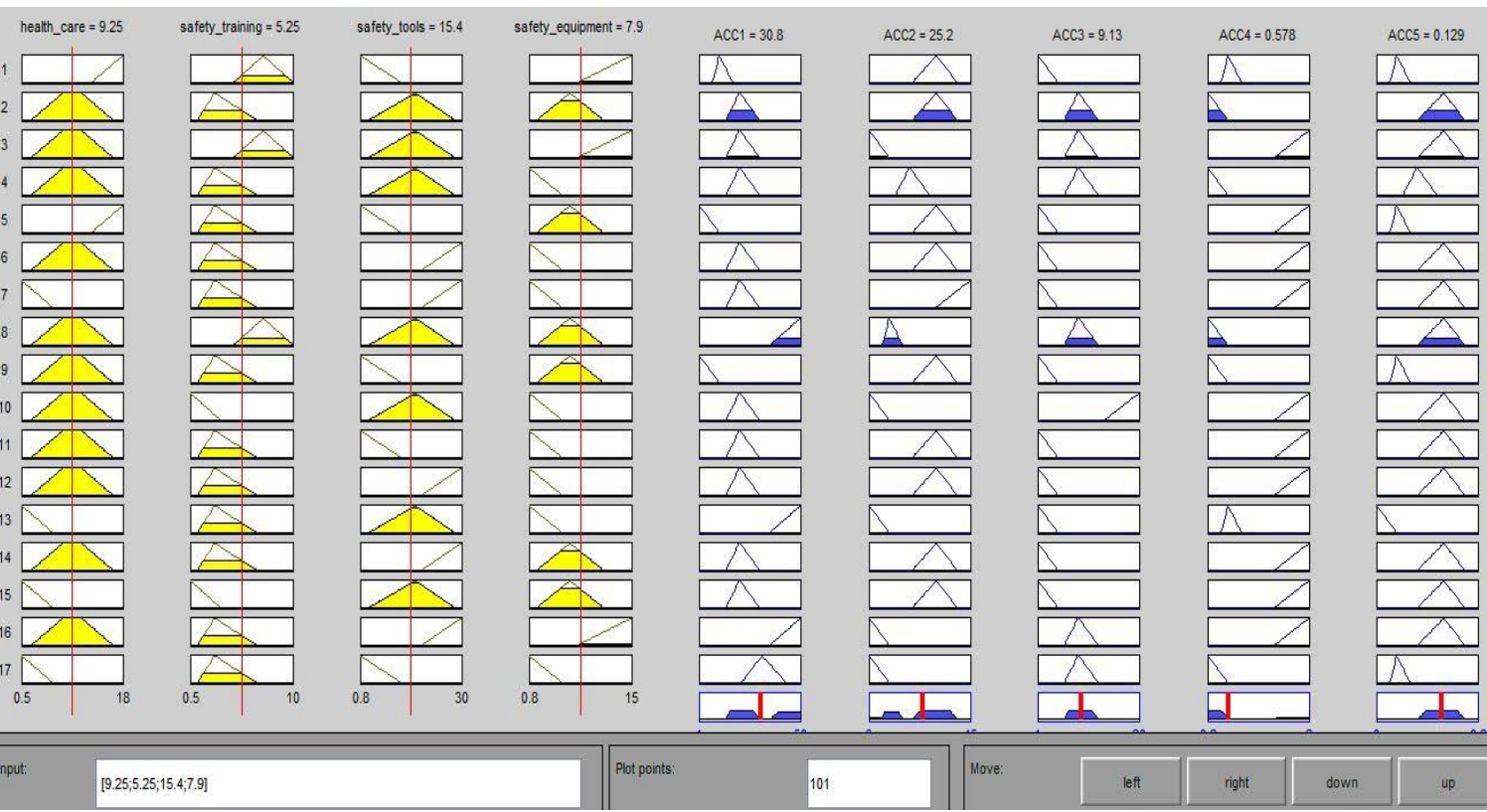

Fig. 8. Rule base obtained after the cognitive intervention.

Table 3. Numerical values of accidents per year (ACC1).

\begin{tabular}{|l|l|l|l|}
\hline Serial no & Actual ACC1(a) & Predicted (p) & Percentage error \\
\hline
\end{tabular}

\begin{tabular}{|c|c|c|c|c|}
\hline IND 1 & 32.6 & 25.5 & 21.77 & 7.1 \\
\hline IND 2 & 30 & 21.9 & 27 & 8.1 \\
\hline IND 3 & 23 & 27.3 & -18.69 & -4.3 \\
\hline IND 4 & 10.5 & 14.1 & -34.28 & -3.6 \\
\hline IND 5 & 26.3 & 32.1 & -22.05 & -5.8 \\
\hline IND 6 & 43.2 & 32.2 & 25.46 & 11 \\
\hline IND7 & 29 & 22.1 & 23.79 & 6.9 \\
\hline IND 8 & 28 & 21 & 25 & 7 \\
\hline IND 9 & 35 & 29.4 & 16 & 5.6 \\
\hline IND 10 & 13 & 10.1 & 22.30 & 2.9 \\
\hline
\end{tabular}


Table 4. Numerical values of accidents per year (ACC2).

\begin{tabular}{|c|c|c|c|c|}
\hline Serial no & Actual ACC2(a) & Predicted (p) & Percentage error & Residual (a-p) \\
\hline IND 1 & 19.7 & 24 & -21.82 & -4.3 \\
\hline IND 2 & 7.5 & 6.09 & 18.8 & 1.41 \\
\hline IND 3 & 22.8 & 26.9 & -17.98 & -4.1 \\
\hline IND 4 & 37.3 & 28.2 & 24.39 & 9.1 \\
\hline IND 5 & 8.6 & 11 & -27.90 & -2.4 \\
\hline IND 6 & 9 & 6.99 & 22.33 & 2.01 \\
\hline IND7 & 5 & 6.49 & -29.8 & -1.49 \\
\hline IND 8 & 33.3 & 30.5 & 8.40 & 2.8 \\
\hline IND 9 & 10 & 12.7 & -27 & -2.7 \\
\hline IND 10 & 27 & 30.4 & -12.59 & -3.4 \\
\hline
\end{tabular}

Table 5. Numerical values of accidents per year (ACC3). \begin{tabular}{|l|l|l|l|l|}
\hline Serial no & Actual ACC3(a) & Predicted (p) & Percentage error & Residual (a-p) \\
\hline
\end{tabular}

\begin{tabular}{|c|c|c|c|c|}
\hline IND 1 & 8.3 & 10.5 & -26.50 & -2.2 \\
\hline IND 2 & 20 & 17.6 & 12 & 2.4 \\
\hline IND 3 & 7.4 & 5.3 & 28.37 & 2.1 \\
\hline IND 4 & 5 & 4.26 & 14.8 & 0.74 \\
\hline IND 5 & 8 & 8.65 & -8.12 & -0.65 \\
\hline IND 6 & 10 & 9.17 & 8.3 & 0.83 \\
\hline IND7 & 10 & 9.14 & 8.6 & 0.86 \\
\hline IND 8 & 12.3 & 8.73 & 29.02 & 3.57 \\
\hline IND 9 & 12 & 8.45 & 29.58 & 3.55 \\
\hline IND 10 & 5.5 & 4.5 & 18.18 & 1 \\
\hline
\end{tabular}

Table 6. Numerical values of accidents per year (ACC4). \begin{tabular}{|l|l|l|l|l|}
\hline Serial no & Actual ACC4(a) & Predicted (p) & Percentage error & Residual (a-p) \\
\hline
\end{tabular}

\begin{tabular}{|c|c|c|c|c|}
\hline IND 1 & 1 & 1.1 & -10 & -0.1 \\
\hline IND 2 & 1.5 & 1.77 & -18 & -0.27 \\
\hline IND 3 & 2 & 1.55 & 22.5 & 0.45 \\
\hline IND 4 & 1 & 0.698 & 30.2 & 0.302 \\
\hline IND 5 & 0.8 & 0.562 & 29.75 & 0.238 \\
\hline IND 6 & 0.5 & 0.371 & 25.8 & 0.129 \\
\hline IND7 & 2 & 1.74 & 13 & 0.26 \\
\hline IND 8 & 0.5 & 0.326 & 34.8 & 0.174 \\
\hline IND 9 & 1 & 0.63 & 37 & 0.37 \\
\hline IND 10 & 1 & 0.783 & 21.7 & 0.217 \\
\hline
\end{tabular}

Table 7. Numerical values of accidents per year (ACC5). \begin{tabular}{|c|c|c|c|c|}
\hline Serial no & Actual ACC5(a) & Predicted (p) & Percentage error & Residual (a-p) \\
\hline
\end{tabular}

\begin{tabular}{|c|c|c|c|c|}
\hline IND 1 & 0.15 & 0.1 & 33.33 & 0.05 \\
\hline IND 2 & 0.2 & 0.129 & 35.5 & 0.071 \\
\hline IND 3 & 0.1 & 0.129 & -29 & -0.03 \\
\hline IND 4 & 0 & 0.0731 & Nil & -0.073 \\
\hline IND 5 & 0 & 0.0589 & Nil & -0.059 \\
\hline IND 6 & 0 & 0.047 & Nil & -0.047 \\
\hline IND7 & 0.2 & 0.128 & 36 & 0.072 \\
\hline IND 8 & 0 & 0.126 & Nil & -0.13 \\
\hline IND 9 & 0.1 & 0.0691 & 30.9 & 0.031 \\
\hline IND 10 & 0.1 & 0.0755 & 24.5 & 0.025 \\
\hline
\end{tabular}




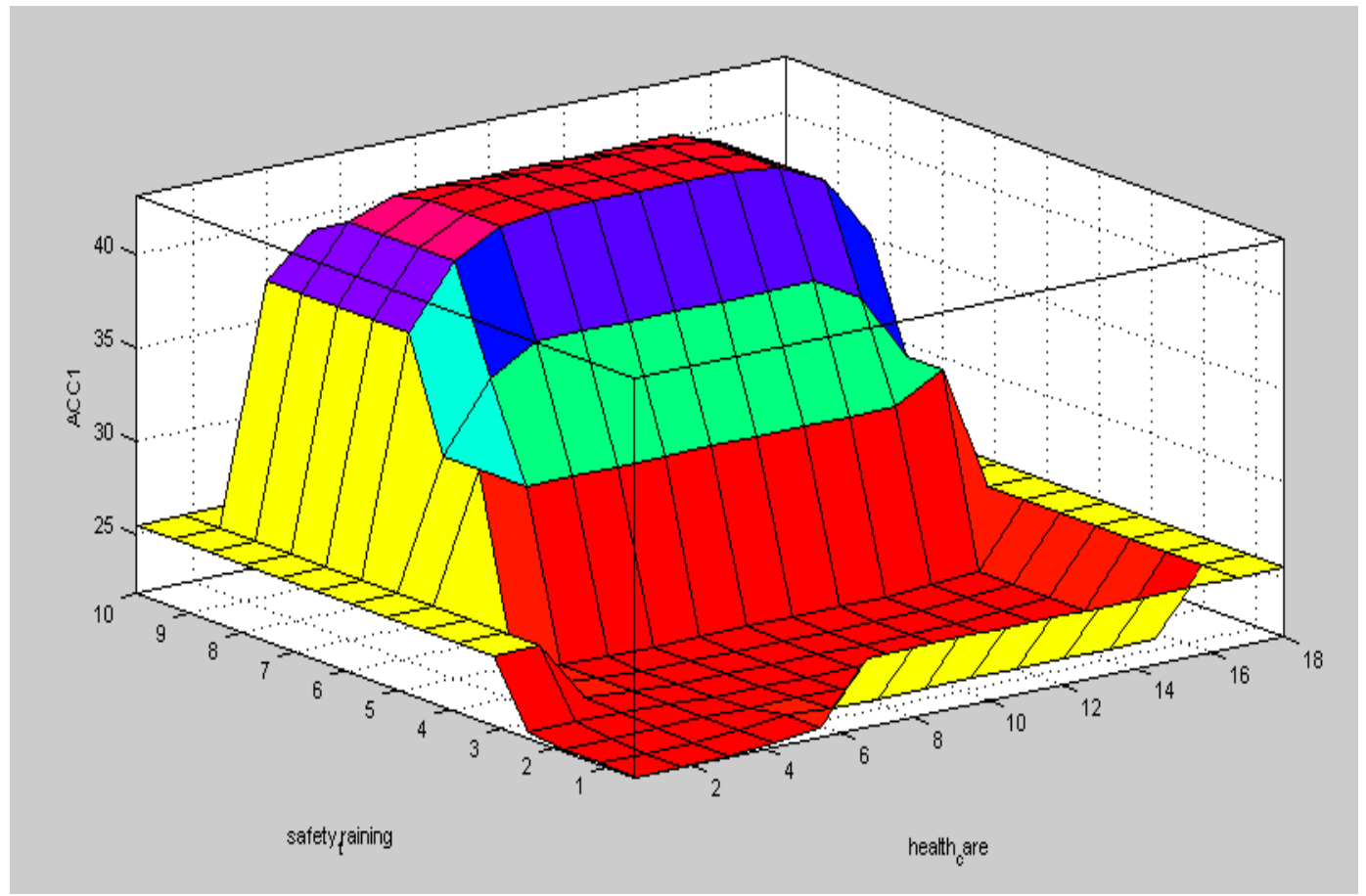

Fig. 9. A surface viewer (output ACC1).

\section{CONCLUSION}

Occupational safety and health (OSH) or workplace health and safety (WHS) deals with the health, safety, and welfare of people involved in work or employment. OSH may also protect family members, employers and customers who might be affected by the workplace environment. Thus, this is a major issue for every industry. Unfortunately, in countries like Bangladesh this is one of the least emphasized concerns which in turn results in a massive loss of life each year along with uncountable injuries. The paper aims to develop an effective tool that predicts accidents in different industries. Management will find it convenient enough as a tool that will help them to make decisions regarding the expenses in health and safety sectors. This will also effectively moderate the number of accidents and injuries of the industry. This model based on fuzzy logic can efficaciously manipulate imprecise data and give an unswerving outcome. The simultaneous use of expert opinion and industrial data makes the decisions more precise.

\section{REFERENCES}

[1] M.F. Chowdhury, T.R. Tanim. Industrial Accidents in Bangladesh Apparel. 2016.

[2] A. Azadeh, I.M. Fam, M. Khoshnoud, M. Nikafrouz. Design and implementation of a fuzzy expert system for performance assessment of an integrated health, safety, environment (HSE) and ergonomics system: the case of a gas refinery, Inform Sci. 2008; 178(22): 4280300p.

[3] G. Beriha, B. Patnaik, S. Mahapatra, S. \& Padhee. Assessment of safety performance in Indian industries using fuzzy approach, Expert Syst Appl. 2012; 39(3): 3311-23p.

[4] M. Dağdeviren, İ. Yüksel. Developing a fuzzy analytic hierarchy process (AHP) model for behavior-based safety management, Inform Sci. 2008; 178(6): 1717-33p.

[5] A. Grassi, R. Gamberini, C. Mora, B. Rimini. A fuzzy multi-attribute model for risk evaluation in workplaces, Saf Sci.2009; 47(5): 707-16p. 
[6] A. Jamshidi, A. Yazdani-Chamzini, S.H. Yakhchali, S. Khaleghi. Developing a new fuzzy inference system for pipeline risk assessment, $J$ Loss Prev Process Ind. 2013; 26(1): 197-208p.

[7] S. Mahdevari, K. Shahriar, A. Esfahanipour. Human health and safety risks management in underground coal mines using fuzzy TOPSIS, Sci Total Environ. 2014; 488: 85-99p.

[8] J. Tah, V. Carr. A proposal for construction project risk assessment using fuzzy logic, Const Manag Econ. 2000; 18(4): 491-500p.
[9] C. Tam, T.K. Tong, G.C. Chiu, I.W. Fung. Non-structural fuzzy decision support system for evaluation of construction safety management system, Int J Project Manag. 2002; 20(4): 303-13p.

[10] L.A. Zadeh. Fuzzy sets, Inform Control. 1965; 8(3): 338-53p.

[11] G. Zheng, N. Zhu, Z. Tian, Y. Chen, B. Sun. Application of a trapezoidal fuzzy AHP method for work safety evaluation and early warning rating of hot and humid environments, Saf Sci. 2012; 50(2): 228-39p. 